\title{
A review of pathophysiology and neuropsychiatric manifestations of COVID-19
}

\author{
Madhu Jasti $^{1}$ (D) Krishna Nalleballe ${ }^{2} \cdot$ Vasuki Dandu $^{2} \cdot$ Sanjeeva Onteddu ${ }^{2}$
}

Received: 28 April 2020 / Revised: 21 May 2020 / Accepted: 25 May 2020 / Published online: 3 June 2020

c) Springer-Verlag GmbH Germany, part of Springer Nature 2020

\begin{abstract}
Introduction The outbreak of coronavirus disease 2019 (COVID-19) has become one of the most serious pandemics of the recent times. Since this pandemic began, there have been numerous reports about the COVID-19 involvement of the nervous system. There have been reports of both direct and indirect involvement of the central and peripheral nervous system by the virus.

Objective To review the neuropsychiatric manifestations along with corresponding pathophysiologic mechanisms of nervous system involvement by the COVID-19.

Background Since the beginning of the disease in humans in the later part of 2019, the coronavirus disease 2019 (COVID19) pandemic has rapidly spread across the world with over 2,719,000 reported cases in over 200 countries [World Health Organization. Coronavirus disease 2019 (COVID-19) situation report-96.,]. While patients typically present with fever, shortness of breath, sore throat, and cough, neurologic manifestations have been reported, as well. These include the ones with both direct and indirect involvement of the nervous system. The reported manifestations include anosmia, ageusia, central respiratory failure, stroke, acute inflammatory demyelinating polyneuropathy (AIDP), acute necrotizing hemorrhagic encephalopathy, toxic-metabolic encephalopathy, headache, myalgia, myelitis, ataxia, and various neuropsychiatric manifestations. These data were derived from the published clinical data in various journals and case reports.

Conclusion The neurological manifestations of the COVID-19 are varied and the data about this continue to evolve as the pandemic continues to progress.
\end{abstract}

Keywords Neuropsychiatric $\cdot$ COVID-19 $\cdot$ Pathophysiology $\cdot$ Stroke $\cdot$ Hyperinflammation

\section{Introduction}

At the end of 2019, many unexplained pneumonia cases occurred in Wuhan, China, and rapidly spread to other parts of China, then to other parts of Asia, Europe, and recently to North America. Eventually, this outbreak was confirmed to be caused by a novel coronavirus [2]. This novel coronavirus reportedly had symptoms resembling that of Severe Acute Respiratory Syndrome Corona Virus (SARS-CoV) seen in the year 2003 [3]. Both these viruses share almost $70 \%$ of the amino acid sequences and use the same receptor-which

Madhu Jasti

madhu.jasti@umm.edu

1 Department of Neurology, University of Maryland Baltimore Washington Medical center, Glen Burnie, USA

2 Department of Neurology, University of Arkansas for Medical Sciences, Little Rock, USA is angiotensin-converting enzyme 2 (ACE2) [4] to gain entry into the cells. Hence, this virus was named SARS-CoV-2. In February 2020, the World Health Organization named the disease as coronavirus disease 2019 (COVID-19).

Coronaviruses cause multiple systemic infections affecting various organ systems, but primarily affect the respiratory system. They tend to mutate and adapt quickly to cross the species barrier, which occurred with SARS$\mathrm{CoV}$ and Middle East respiratory syndrome coronavirus (MERS-CoV), causing epidemics and pandemics. Infection with these viruses in humans often leads to severe clinical symptoms with high mortality [5]. All three of these novel viruses (SARS-CoV, SARS-CoV-2/COVID-19, and MERS-CoV) originate from zoonotic transmission. These are enveloped, positive-stranded RNA corona viruses in the betacoronoviride family. Studies had demonstrated that the clinical course of SARS and MERS was highly similar and SARS and MERS may have similar pathogenesis [6]. The 
genome sequence of SARS-CoV-2 also shows some similarities to that of MERS-CoV. The postulated common pathophysiologic mechanisms include dysregulation of cytokines/ chemokines, deficiencies in the innate immune response, direct infection of immune cells, direct viral cytopathic effects, and autoimmunity [7]. MERS-CoV infects cells by binding to the dipeptidyl peptidase 4 receptor, and SARS$\mathrm{CoV}$ acts via the angiotensin-converting enzyme 2 receptor [8].

As for COVID-19, numerous studies have described typical clinical manifestations including fever, cough, sore throat, shortness of breath, diarrhea, and fatigue. COVID-19 also has characteristic laboratory findings and lung computed tomography abnormalities [9]. However, there is growing literature that patients with COVID-19 have cardiac [10] and neuropsychiatric manifestations, as well. A recently published study that looked at 214 cases of severe coronavirus illness treated in Wuhan during the early phase of the global pandemic reported that about $36 \%$ of patients displayed neurological symptoms [11]. This study reports that strokes, altered consciousness, and other neurological issues are relatively common in serious cases of COVID19. These include both direct and indirect involvement of the nervous system. Following this study, there have been several case reports of various neurologic diagnoses. The reported manifestations include anosmia, ageusia, stroke, AIDP, acute necrotizing hemorrhagic encephalopathy, toxic-metabolic encephalopathy, headache, myalgia, central respiratory failure, myelitis, ataxia, and various neuropsychiatric manifestations.

\section{Discussion}

In this section, we will discuss the neuropsychiatric presentations and the possible associated pathophysiology.

\section{Anosmia and ageusia}

Losses of smell and taste have been strongly linked to COVID-19 infections [12]. So far, there have been numerous publications about the association of loss of smell and taste with COVID-19 infection. Post-viral anosmia is one of the leading causes of loss of sense of smell in adults. This post-viral loss of smell is thought to be secondary to initial congestion of the nose, which leads to loss of fine hair like endings of the olfactory receptor cells causing them to be ineffective in picking up odor molecules from the nose. SARS-CoV-2 also appears to be highly concentrated in the nostrils of affected patients causing inflammation of the olfactory nerves and structural damage to the receptors and thereby causing anosmia [13]. Studies have shown that the tongue has a very high expression of ACE2 receptors compared to buccal and gingival tissues, thereby posing a high risk of viral binding and ageusia from taste receptor damage [14].

\section{Central respiratory failure}

There have been a fair number of reports suggesting SARSCoV-2 infecting the neurons, raising questions about the direct effects of the virus on the brain that play a role in patients' deaths. Some of the respiratory symptoms due to the disease might actually be secondary to respiratory center involvement controlled by the nervous system. According to Yan-Chao Li, et al. [15], SARS-CoV-2 infects nerve cells, particularly the neurons in the medulla oblongata, which serves as the control center for the heart and the lungs. The damage to this area could contribute to the acute respiratory failure of patients with COVID-19. Autopsy results of patients with COVID-19 showed that the brain tissue near brainstem was hyperemic and edematous with neuronal degeneration [16]. By contrast, there have been a few case reports which mention no penetrance of virus into the central nervous system as evidenced by the absence of SARS-CoV-2 in CSF and that the CNS effects are secondary to elevated inflammatory markers as CSF analyses during the acute stage showed pleocytosis with increased IL- 8 and TNF- $\alpha$ concentrations [17].

Researchers have reported that many human cell types express ACE2, including lung, heart, kidney, intestine, and brain tissue [18]. There are at least a couple of ways that the virus could invade the central nervous system-it might circulate through the blood and then attack ACE2 receptors in the endothelia that line blood capillaries in the brain, breaching the blood-brain barrier and invading neurons through that route (Fig. 1). A breached blood-brain barrier could also cause brain swelling, compressing the brain stem there by affecting respiration. Apart from these two mechanisms, it has also been demonstrated that some coronaviruses can spread by synaptic transfer from chemoreceptors and mechanoreceptors in lung to the medullary cardiorespiratory center [19]. This process could be an implicating factor in acute onset respiratory failure in some of the COVID-19 patients [20].

\section{Acute inflammatory demyelinating polyneuropathy, myelitis}

Researchers in China published the first presumptive case of Acute Inflammatory Demyelinating Polyneuropathy (AIDP)/ Guillain-Barre syndrome (GBS) associated with COVID19 on Apr 1, 2020 [21]. Reportedly, the patient initially presented with signs of the autoimmune neuropathy after returning from Wuhan, China and later tested positive for the COVID-19. Considering the temporal association, it 


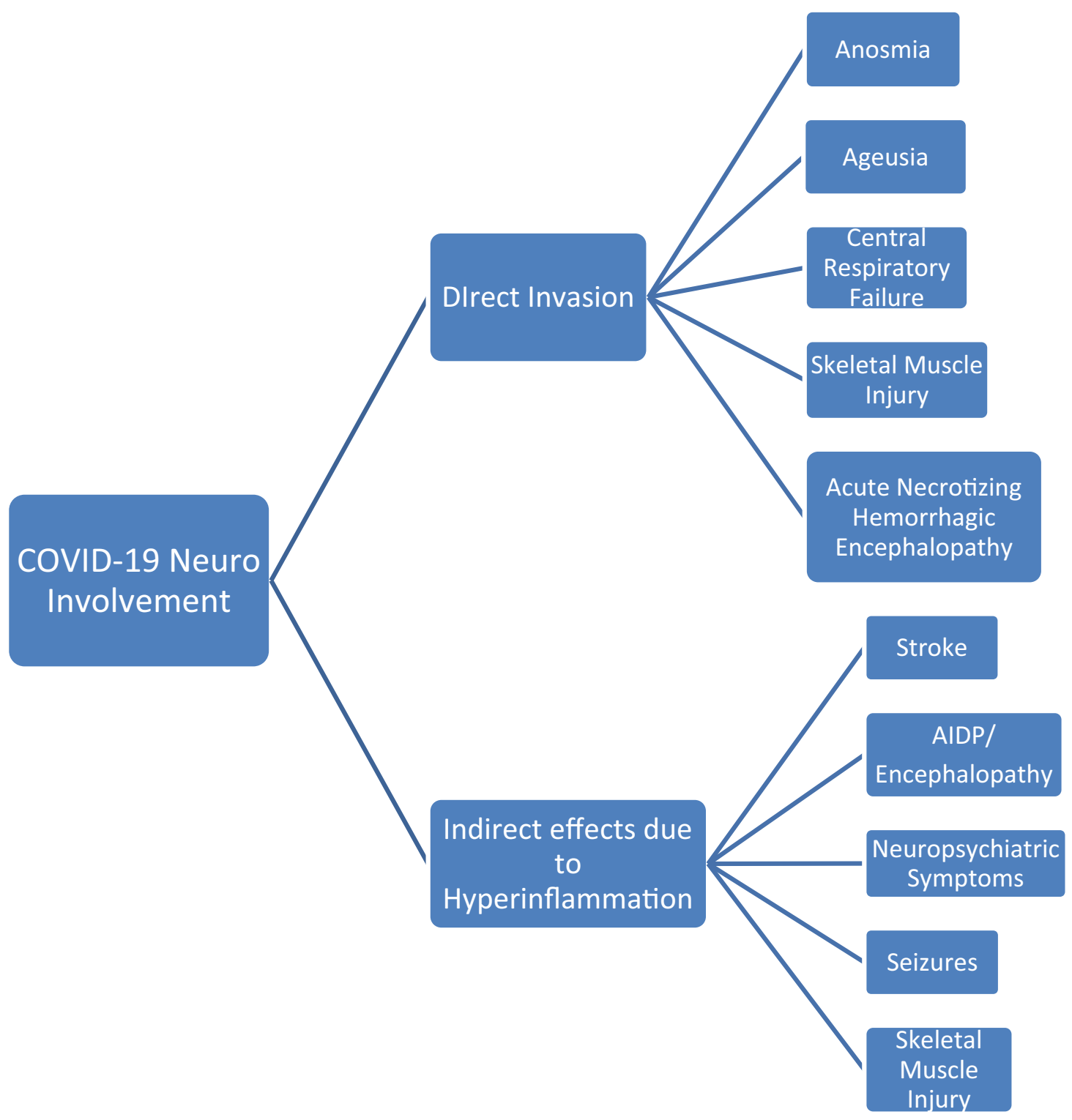

Fig. 1 COVID-19 neuro manifestations

was speculated that SARS-CoV-2 infection might have been responsible for the development of AIDP. Following this, a case series from Italy, published by Toscano et al., reported five cases of AIDP that started after the onset of COVID-19 disease [22]. Around the same time, two case reports were published from Spain reporting the occurrence of Miller Fisher syndrome and polyneuritis cranialis in patients diagnosed with COVID-19 [23]. All these studies showed that AIDP occurs early in the course of the disease and followed the pattern of a para-infectious profile, instead of the classic post-infectious profile.

The underlying pathophysiologic mechanisms might be secondary to the neuroinvasive nature of the virus precipitating demyelination [24] versus viral infection creating an inflammatory environment triggering an aberrant immune response (secondary to molecular mimicry) leading to peripheral demyelination [25].

\section{Acute necrotizing hemorrhagic encephalopathy}

Poyiadji et al. reported the first presumptive case of COVID19-associated acute necrotizing hemorrhagic encephalopathy [26]. Acute necrotizing encephalopathy is a rare encephalopathy and one of the remote complications of influenza and other viral infections. This has been presumed to be due to intracranial cytokine storm, which results in the blood-brain barrier breakdown, without direct viral invasion or para-infectious demyelination [27]. Accumulating 
evidence suggests that a subgroup of patients with severe COVID-19 might have a cytokine storm syndrome [28].

A cytokine profile resembling secondary hemophagocytic lymphohistiocytosis (a hyperinflammatory syndrome that leads to fulminant and fatal hypercytokinaemia with multiorgan failure which is commonly triggered by viral infections [29]) is associated with severe COVID-19, characterized by increased interleukin's-IL-2, IL-7, granulocyte-colony stimulating factor, interferon-alpha, monocyte chemoattractant protein 1, macrophage inflammatory protein 1-alpha, and tumor necrosis factor-alpha [30]. Predictors of fatality from a recent retrospective, multicenter study of 150 confirmed COVID-19 cases in Wuhan, China, included elevated ferritin and IL-6 [31], suggesting that hyper inflammation might be contributing to mortality.

\section{Stroke}

Patients with severe infection were more likely to develop neurologic manifestations, especially acute cerebrovascular disease. Patients with severe infection had higher D-dimer levels than that of patients with the non-severe infection, which may be the reason why patients with severe infection are more likely to develop the cerebrovascular disease. Apart from the elevated d-dimer causing a state of altered coagulation cascade, there is also a theory that there is a vasculitis type of picture created secondary to intracranial cytokine storm versus the infection by the virus itself which are believed to be the possible pathophysiologic mechanisms behind stroke/cerebrovascular accident [32]. The virus can get access to cerebral circulation from systemic circulation, attach to endothelium ACE2 receptors, and cause endothelial ruptures and thrombus [33]. Slow cerebral circulation compared to systemic circulation pose an increased risk of replication and rupture. Also, the virus can get into brain tissue from capillary endothelium by brain-blood barrier disruption and cause neuronal damage without much inflammation (Fig. 1).

\section{Neuropsychiatric symptoms}

Past studies on viral pandemics, especially involving respiratory viruses, suggest that diverse types of neuropsychiatric symptoms can arise with acute infection as well as in the post-viral infectious period [34]. One study reported persistent neurocognitive deficits up to 18 months post-discharge [35]. In the acute phase, apart from being the psychosocial stressor, COVID-19 has been reported to cause neuropsychiatric manifestations, like encephalopathy, psychosis, insomnia, and mood changes. Post-traumatic stress disorder, panic attacks, anxiety are mostly seen in health care workers and survivors of SARS CoV infection [36]. This is largely secondary to the mental trauma and not as a direct consequence of infection. In addition, over-reactive behavior due to fear is usually noted in the public during the pandemics [37]. Aggression, frustration, can worsen with quarantine and lockout procedures [38].

These neuropsychiatric manifestations have been attributed to viral infection per se and also secondary to the host immune response [39]. Direct viral infiltration of the central nervous system can trigger a neuro-inflammatory reaction leading to microglial activation [40], which in turn triggering demyelinating processes is one of the primary etiologies for encephalopathy. In the absence of direct viral infiltration, peripheral hypercytokinaemia causing an imbalance of neurotransmitters within the central nervous system has been implicated in neuropsychiatric manifestations. The state of hypercytokinaemia triggers a neuro-inflammatory response causing disruption of the blood-brain barrier, leading to peripheral immune cell transmigration into the central nervous system and, in turn, causing imbalances in neurotransmission [41].

\section{Skeletal muscle injury and myalgia}

ACE2 was identified as the functional receptor to enter into a cell for SARS-CoV-2, which is present in multiple human organs, including the nervous system and skeletal muscles. Patients with severe COVID-19 disease had muscle breakdown causing muscle weakness and this manifested as elevated creatine kinase and lactate dehydrogenase levels than those without muscle symptoms. Apart from the direct tissue injury by the virus, cytokine storm damage [42] might also be the other reason for muscle involvement with COVID-19.

\section{Ataxia}

This has been commonly reported in patients with moderately severe disease. The pathophysiologic mechanisms are unclear, but could be secondary to breached blood-brain barrier and involvement of the brain stem versus elevated inflammatory markers.

\section{Toxic-metabolic encephalopathy}

Patients with severe disease had a prolonged ICU course and were noted to be encephalopathic for more than the usually expected duration. This is most likely secondary to the use of multiple and high doses of anesthetics and sedatives as a part of the symptomatic management of severe respiratory disease. Hypoxia and viremia itself are also the possible factors behind encephalopathy [43]. 


\section{Seizures, headaches}

Headache was commonly seen in patients with mild-tomoderate disease severity. These were partly believed to be secondary to raised inflammatory mediators in the body and decreased cerebral blood flow from hypoxia and endothelial changes from viremia. Seizures were also randomly reported. These have been hypothesized to be secondary to decreased seizure threshold secondary to an innate immune response from cytokine surge [44] rather than viruses primarily causing the seizures.

\section{Challenges of nervous system involvement}

Dense parenchyma and imperviousness of brain tissues not only protects the brain from infectious processes, but also poses a challenge to eliminate them once brain involvement occurs [45]. Cytotoxic $\mathrm{T}$ cells are the mainstay for the elimination of viruses from brain tissue because of the lack of major histogen compatibility antigens in neurons 46. Therefore, more research about COVID-19 neurogenic involvement is necessary to identify and treat the neurological disease early, rather than at an advanced stage at which it will be more challenging.

\section{Conclusions}

Patients with COVID-19 commonly have neurologic manifestations. The data/literature on this continues to evolve. On one hand, there are very commonly reported neurologic diagnoses of anosmia, encephalopathy, and stroke, and at the same time, there have been only a few isolated case reports of acute necrotizing encephalopathy and AIDP. We have to keep in mind that the current information that we have about the neurologic manifestations of COVID-19 is in the context of purposefully avoiding advanced neurodiagnostic procedures like magnetic resonance imaging, lumbar puncture, electromyography, and nerve conduction studies to reduce the risk of crossinfection within the hospital. A prospective, observational study with a larger number of patients that includes more specific neuro-imaging and other diagnostic tests is warranted for more conclusive evidence.

\section{Compliance with ethical standards}

Conflicts of interest No conflicts of interest.

\section{References}

1. World Health Organization. Coronavirus disease 2019 (COVID19) situation report-96.

2. Zhu N, Zhang D, Wang W et al (2020) China novel coronavirus investigating and research team a novel coronavirus from patients with pneumonia in China. N Engl J Med. 382(8):727-733

3. Zhou P, Yang XL, Wang XG et al (2020) A pneumonia outbreak associated with a new coronavirus of probable bat origin. Nature 579(7798):270-273

4. Zhao Y, Zhao Z, Wang Y, Zhou Y, Ma Y, Zuo W (2020) Singlecell RNA expression profiling of ACE2, the putative receptor of Wuhan 2019-nCov. bioRxiv 10:12

5. World Health Organization. Middle East respiratory syndrome coronavirus (MERS-CoV). Published November 2019.

6. De Wit, van Doremalen, Falzarano, Munster (2016) SARS and MERS: Recent insights into emerging coronaviruses. Nat Rev Microbiol. 14:523

7. Jiang Gu, Korteweg C (2007) Pathology and pathogenesis of severe acute respiratory syndrome. Am J Pathol 170(4):1136-1147

8. Cui J, Li F, Shi ZL (2019) Origin and evolution of pathogenic coronaviruses. Nat Rev Microbiol 17(3):181-192

9. Guan WJ, Ni ZY, Hu Y et al (2020) China medical treatment expert group for Covid-19. Clinical characteristics of coronavirus disease 2019 in China. N Engl J Med 382:1708-1720

10. Siddamreddy S, Thotakura R, Dandu V, Disease CV et al (2019) (COVID-19) Presenting as acute ST elevation myocardial infarction. Cureus 12(4): 7782

11. Mao L, Jin H, Wang $M$ et al. Neurologic manifestations of hospitalized patients with coronavirus disease 2019 in Wuhan, China. JAMA Neurol. Published online April 10, 2020.

12. Shima T. Moein et al. Smell dysfunction: a biomarker for COVID-19. alr.22587

13. Baig AM, Khaleeq A, Ali U, Syeda H (2020) Evidence of the COVID-19 virus targeting the CNS: tissue distribution, hostvirus interaction, and proposed neurotropic mechanisms. ACS Chem Neurosci 11(7):995-998

14. Xu H, Zhong L, Deng J et al (2020) High expression of ACE2 receptor of 2019-nCoV on the epithelial cells of oral mucosa. Int J Oral Sci 12(1):8

15. Yan-Chao L, Wan-Zhu B, Hashikawa T (2020) The neuroinvasive potential of SARS-CoV2 may play a role in the respiratory failure of COVID-19 patients. J Med Virol. https://doi. org/10.1002/jmv. 25728

16. National Health Commission of the People's Republic of China. Diagnosis and treatment of the novel coronavirus pneumonia. Published 2020.

17. Pilotto A et al (2020) Steroid-responsive Encephalitis in Covid19 Disease. Ann Neurol. https://doi.org/10.1002/ana.25783./ jmv. 25728

18. Hamming I, Timens W, Bulthuis ML, Lely AT, Navis G, van Goor H (2004) Tissue distribution of ACE2 protein, the functional receptor for SARS coronavirus: a first step in understanding SARS pathogenesis. J Pathol. 203(2):631-637

19. Umapathi T, Kor AC, Venketasubramanian N et al (2004) Large artery ischaemic stroke in severe acute respiratory syndrome (SARS). J Neurol 251(10):1227-1231

20. Li YC, Bai WZ, Hashikawa T (2020) The neuroinvasive potential of SARS-CoV2 may play a role in the respiratory failure of COVID-19 patients. J Med Virol. https://doi.org/10.1002/ jmv. 25728

21. Zhao H et al (2020) Guillain-Barré syndrome associated with SARS-CoV-2 infection: causality or coincidence? Lancet Neurology published Apr 1, 2020 
22. Gianpaolo Toscano et al (2020) Guillain-Barré Syndrome Associated with SARS-CoV-2. NEJM. https://doi.org/10.1056/NEJMc 2009191

23. Consuelo Gutierrez et al (2020) Miller Fisher syndrome and polyneuritis cranialis in COVID-19. Neurology. https://doi. org/10.1212/WNL.0000000000009619

24. Hosking MP, Lane TE (2010) The role of chemokines during viral infection of the CNS. PLoS Pathog 6(7):e1000937

25. Fairweather D, Frisancho-Kiss S, Rose NR (2005) Viruses as adjuvants for autoimmunity: evidence from Coxsackievirusinduced myocarditis. Rev Med Virol 15:17-27

26. Poyiadji N et al (2020) COVID-19-associated acute hemorrhagic necrotizing encephalopathy: CT and MRI features. Images Radiol. https://doi.org/10.1148/radiol.2020201187

27. Rossi A (2020) Imaging of acute disseminated encephalomyelitis. Neuroimaging Clin 18(1):149-161

28. Mehta P, McAuley DF, Brown M et al (2020) COVID-19: consider cytokine storm syndromes and immunosuppression. Lancet 395(10229):1033-1034

29. Ramos-Casals M et al (2014) Adult haemophagocytic syndrome. Lancet 383:1503-1516

30. Huang $\mathrm{C}$ et al (2020) Clinical features of patients infected with 2019 novel coronavirus in Wuhan. China Lancet 395:497-506

31. Ruan Q et al. (2020) Clinical predictors of mortality due to COVID-19 based on an analysis of data of 150 patients from Wuhan, China. Intensive Care Med 46(5):846-848

32. Oxley TJ, MD, et al (2020) Large-vessel stroke as a presenting feature of Covid-19 in the young. N Engl J Med 382:e60

33. Akshay Avula et al (2020) COVID-19 presenting as stroke. Brain Behav Immun. https://doi.org/10.1016/j.bbi.2020.04.077

34. Honigsbaum M (2013) The art of medicine: "an inexpressible dread": psychoses of influenza at fin-de-siècle. Lancet 381:988-989

35. Salluh JIF, Wang H, Schneider EB, Nagaraja N, Yenokyan G, Damluji A, Serafim RB, Stevens RD (2015) Outcome of delirium in critically ill patients: systematic review and meta-analysis. BMJ 350:1-10
36. Gardner PJ, Moallef P (2015) Psychological impact on SARS sur- vivors: critical review of the English language literature. Can Psychol/Psychol Canadienne 56:123

37. Person B, Sy F, Holton K, Govert B, Liang A (2004) Fear and stigma: the epidemic within the SARS outbreak. Emerg Infect Dis $10: 35$

38. Xiang YT, Yang Y, Li W, Zhang L, Zhang Q, Cheung T, Ng CH (2020) Timely mental health care for the 2019 novel corona virus outbreak is urgently needed. Lancet

39. Troyer EA, Kohn JN, Hong S (2020) Are we facing a crashing wave of neuropsychiatric sequelae of COVID-19? Neuropsychiatric symptoms and potential immunologic mechanisms. Brain Behav. https://doi.org/10.1016/j.bbi.2020.04.027

40. Wohleb ES, McKim DB, Sheridan JF, Godbout JP (2015) Monocyte trafficking to the brain with stress and inflammation: a novel axis of immune-to-brain communication that influences mood and behavior. Front Neurosci 9:1-17

41. Dantzer R (2018) Neuroimmune interactions: From the brain to the immune system and vice versa. Physiol Rev 98:477-504

42. Fadila MF, Wool KJ (2015) Rhabdomyolysis secondary to influenza a infection: a case report and review of the literature. $\mathrm{N}$ Am J Med Sci 7:122-124

43. Wu Y, Xu X, Chen Z, et al. (2020) Nervous system involvement after infection with COVID-19 and other coronaviruses. Brain Behav Immun

44. Libbey JE, Kennett NJ, Wilcox KS, White HS, Fujinami RS (2011) Interleukin-6, produced by resident cells of the central nervous system and infiltrating cells, contributes to the development of seizures following viral infection. J Virol 85(14):6913-6922

45. Reinhold AK, Rittner HL (2017) Barrier function in the peripheral and central nervous system-a review. Pflugers Arch 469(1):123-134

46. Wuthrich C, Batson S, Koralnik IJ (2015) Lack of major histocompatibility complex class I upregulation and restrictive infection by $\mathrm{JC}$ virus hamper detection of neurons by T lymphocytes in the central nervous system. J Neuropathol Exp Neurol 74(8):791-803 